\title{
Micro-imaging and Microanalysis of PLGA Complex Drug Products by Scanning Electron Microscopy (SEM)
}

\author{
Jing Liang ${ }^{1}$, Yan Wang ${ }^{1}$, Bin Qin ${ }^{1}$, Jia Zhou ${ }^{2}$, Steven Schwendeman ${ }^{2}$ and Jiwen Zheng ${ }^{1}$
}

${ }^{1}$ Food and Drug Administration, Silver Spring, Maryland, United States, ${ }^{2}$ University of Michigan, Ann Arbor, Michigan, United States

\section{Introduction}

Due to its outstanding biocompatibility and biodegradability, poly(lactide-co-glycolide) (PLGA) has been extensively used in FDA-approved long acting drug products (e.g., microspheres and implants). Despite the vast demand, there is no generic PLGA drug products on the U.S. market until now [1]. One of the reasons is the complexity associated with formulation design and physicochemical characterization. It has been well recognized that microstructure/porosity of PLGA matrix plays an important role on drug release and PLGA degradation. However, determining porosity and inner microstructure is often challenging. The goal of the current project was to explore using regular room temperature scanning electron microscope (SEM) and cryo-SEM to characterize both external and internal morphology of in house prepared PLGA microspheres containing leuprolide acetate and a commercial product (Lupron Depot®, $7.5 \mathrm{mg}$ ).

\section{Methods}

Both room temperature SEM and cryogenic -SEM (cryo-SEM) coupled with energy dispersive X-ray spectrometer (EDS) were used to characterize commercially available brand name product, Lupron Depot ${ }^{\circledR}$ microspheres, together with in-house PLGA microsphere formulations. The leuprolide acetate encapsulated PLGA microspheres were prepared as described before [2]. To study their external structures, Lupron Depot ${ }^{\circledR}$ microspheres and in-house PLGA microspheres were placed on conductive tape while their internal structures were exposed after cutting using a razor blade. The samples were then sputtered with $5 \mathrm{~nm}$ gold before imaging by SEM (TESCAN Mira3) at room temperature. The microspheres were also suspended in phosphate buffered saline (PBS) buffer and then were cryo-fixed with a high-pressure freezer (Leica ICE) to preserve their native structures [3, 4]. Cross-sectional specimens were prepared by freeze-fracture (Leica ACE 600). After freeze fracture, the samples were transferred to SEM chamber using a cryo-transfer system (Leica VCT 500) for imaging at $-145^{\circ} \mathrm{C}$. EDS (Oxford Max-80 SDD) microanalysis was performed to identify the distribution of PLGA and water.

\section{Results}

The typical external morphology of Lupron Depot ${ }^{\circledR}$ microspheres and in-house PLGA microspheres was showed in Fig.1A and Fig.1B, respectively. Compared to Lupron Depot ${ }^{\circledR}$, the in-house prepared PLGA microspheres had a more porous external structure, which provides a higher surface area in contact with releasing medium. This structure difference was further confirmed by examining the cross-section of microspheres. The cross-section of Lupron Depot ${ }^{\circledR}$ microspheres in Fig.1C showed uniformly condensed structure while the in-house PLGA microspheres in Fig.1D demonstrated a core-shell structure where the core was dense, and the shell was more porous. Despite the information revealed by room temperature SEM regarding the external and internal morphology, the pore connectivity can only be visualized under hydrated condition. Cryo-SEM could provide this information readily. Fig. 2 showed cross-section of the Lupron Depot ${ }^{\circledR}$ (left) and in-house PLGA microspheres (right) dispersed in PBS together with EDS line scan of carbon and oxygen along the yellow line. The PLGA contains $\mathrm{C}, \mathrm{H}$ and $\mathrm{O}$, while water only contains $\mathrm{H}$ and $\mathrm{O}$. The $\mathrm{C} / \mathrm{O}$ ratio can be used to demonstrate the polymer and water distribution $[5,6]$. 
The $\mathrm{C} / \mathrm{O}$ ratio mapping showed the Lupron Depot ${ }^{\circledR}$ microspheres had a solid structure while the in-house PLGA microspheres had interconnected pores in the shell part and the core part appeared solid.

\section{Conclusions}

Through room temperature SEM and cryo-SEM, we characterized the external morphology and internal structure of Lupron Depot ${ }^{\circledR}$ microspheres and the in-house PLGA microspheres. While the room temperature SEM can provide enough information about the external structure, the cryo-SEM could provide more comprehensive information about the internal pore structure, especially for connected pores. Cryo-SEM combined with room temperature SEM could serves as powerful tools to determine matrix porosity, which subsequently helps obtain mechanistic understanding on drug release and polymer degradation to facilitate formulation development of PLGA products.

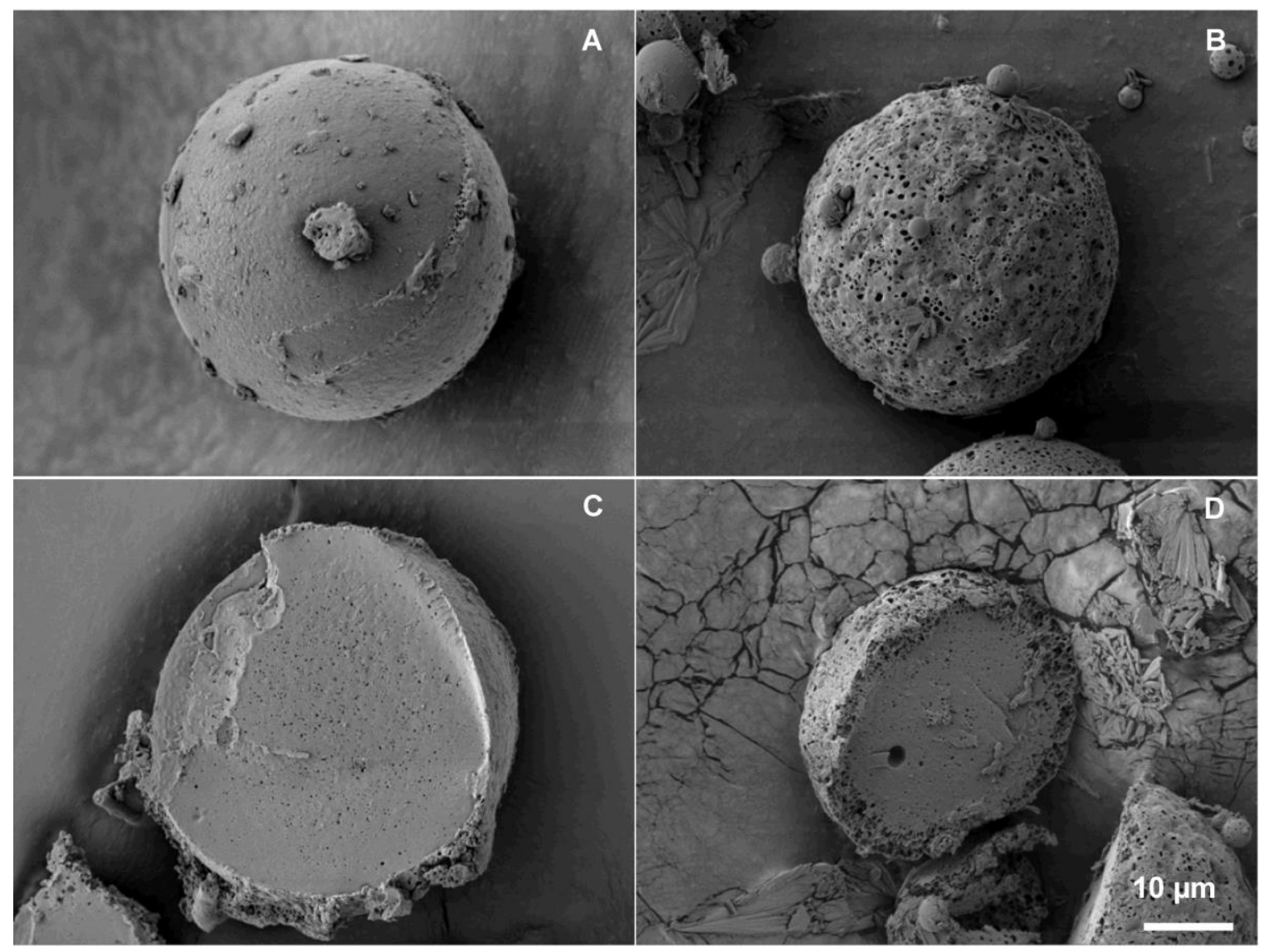

Figure 1. Room temperature SEM images of Lupron Depot ${ }^{\circledR}$ microsphere (A) with its cross-section (C) and in-house formulated PLGA microsphere (B) with its cross-section (D). 

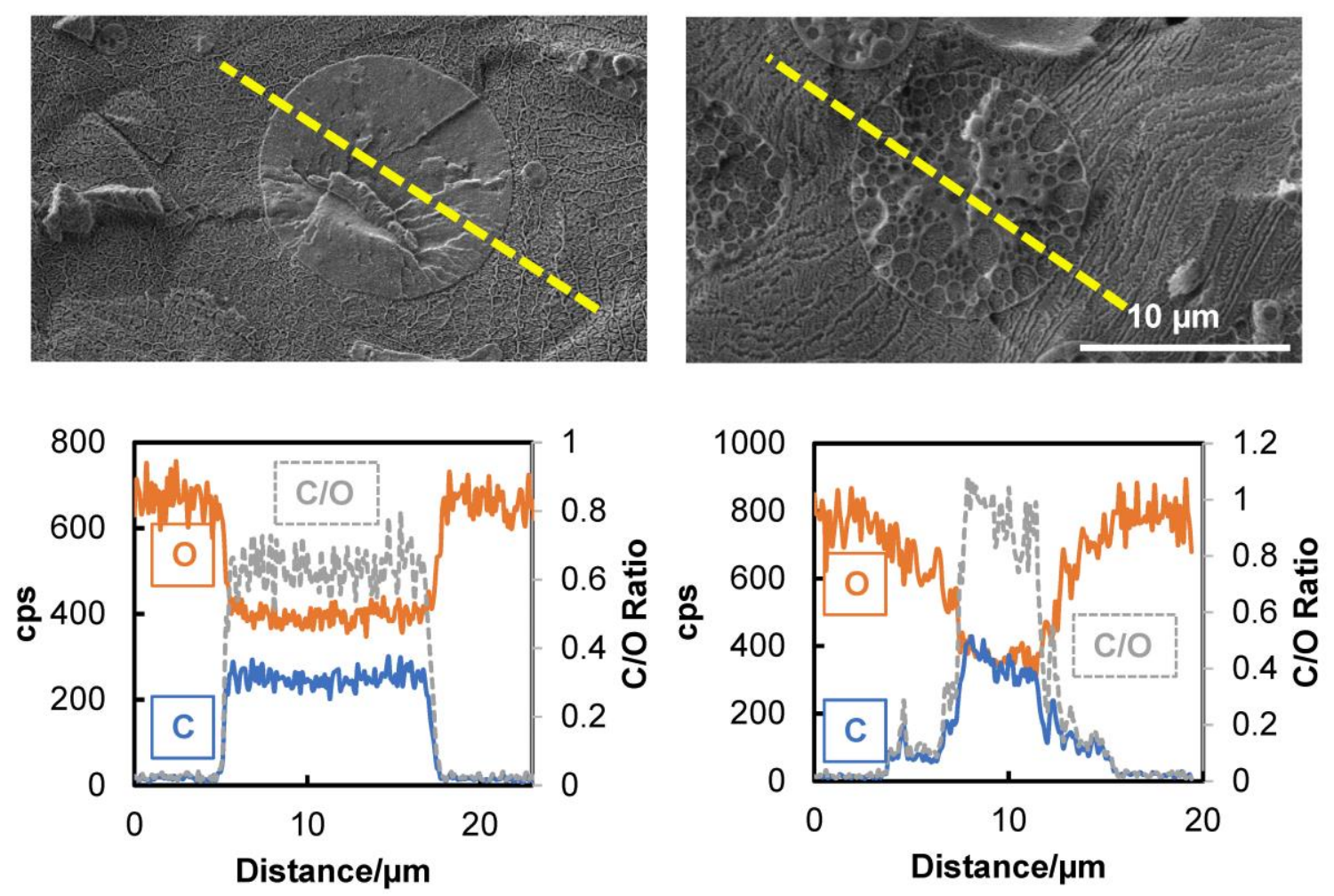

Figure 2. Cryo-SEM image and EDS line scan of Lupron Depot ${ }^{\circledR}$ microsphere (left) and in-house formulated PLGA microsphere (right).

\section{References}

[1] Yan Wang et al, FDA's regulatory science program for generic PLA/PLGA-based drug products, American Pharmaceutical Review, 19(2016), p. 5.

[2] Jia Zhou et al, Effect of manufacturing variables and raw materials on the composition-equivalent PLGA microspheres for 1-month controlled release of leuprolide, Molecular Pharmaceutics, (2020), https://pubs.acs.org/doi/10.1021/acs.molpharmaceut.9b01188

[3] Yong $\mathrm{Wu}$ et al, Extracellular matrix reorganization during cryo preparation for scanning electron microscope imaging of Staphylococcus aureus biofilms, Microscopy and Microanalysis, 20(2014), p. 1348.

[4] Jing Liang et al, Freezing and sublimation effects on cryo-SEM imaging and microanalysis, Microscopy and Microanalysis, 25(2019), p.1108.

[5] Jing Liang et al, Measuring microgel swell ratio by cryo-SEM, Polymer, 116(2017), p. 1.

[6] Jing Liang et al, Counterion exchange in peptide-complexed core-shell microgels, Langmuir, 35(2019), p. 9521. 